\title{
Synthesis of Bifunctional Allylic Compounds using Cyclopropenes as Functionalized Allyl Equivalents
}

\author{
Sergio Mata, Luis A. López, ${ }^{*}$ and Rubén Vicente* \\ Dedicated to our colleague Prof. Francisco J. Fañanás on the occasion of his retirement
}

\begin{abstract}
The synthesis of uncommon bifunctional allylic derivatives bearing a silane and an alcohol within the same allylic framework is reported. This method relies on the coupling of hydrosilanes with substituted and functionalized cyclopropenes, which deliver the allyl fragment. Rhodium(II) catalysts provide regioselective access to vinyl carbene intermediates, which easily undergo $\mathrm{Si}-\mathrm{H}$ bond insertions. The transformation occurs with complete atom economy and shows a remarkable broad scope, including intramolecular version for the synthesis of cyclic O-Si-linked compounds as well as the synthesis of the corresponding allyl amines.
\end{abstract}

Organic synthesis strongly relies on assembling building blocks, small molecules which are commercially offered or synthetically easy-to-prepare. Chemical versatility is an enticing added value for building blocks as this feature allows their uses for diversityoriented synthesis. ${ }^{[1,2]}$ This ability requires the combination of several moieties into a molecule, which generally results in the further need to prepare these building blocks.

Within available synthons in organic synthesis, allylsilanes ${ }^{[3]}$ and allylic alcohol derivatives ${ }^{[4]}$ hold prevalent positions. A plethora of transformations ranging from simple alkene, alcohol or silicon chemistry to more sophisticated metal-allyl chemistry, justifies their synthetic utilities. Interestingly, combining silane and alcohol moieties within the same allyl system might create a bifunctional structure, such as A (Scheme 1, a) with potential uses as a flexible building block. While allylsilanes and allyl alcohols themselves are effortlessly on hand, scaffold $\mathbf{A}$ is far less common. Considering the latent interest of structure $\mathbf{A}$ and relying on previous studies, ${ }^{[5]}$ we devised a simple route for the synthesis of $\mathbf{A}$ through $\mathrm{Si}-\mathrm{H}$ bond carbene insertion. While silyl piece $\mathbf{B}$ is elementary, selection of a carbene-fragment precursor seems less obvious. Yet, using adequately decorated cyclopropene $\mathbf{C},{ }^{[6,7]}$ which can be routinely prepared ${ }^{[8]}$ carbene intermediate $\mathbf{D}$ might be accessible providing access to desired scaffold A (Scheme 1, a). In this manner, a cyclopropene serves as a worthy allyl synthetic equivalent, an underestimated utility of this class of compounds. ${ }^{[9]}$ This strategy faces also important challenges. First, generation of the desired carbene intermediate must occur through a regioselective cyclopropene rearrangement. Then, an intermolecular reaction must outplay several imaginable intra- or intermolecular side-reactions, such

[a] S. Mata, Dr. L. A. López, ${ }^{*}$ Dr. R. Vicente*

Departamento de Química Orgánica e Inorgánica e Instituto Universitario de Química Organometálica "Enrique Moles" Universidad de Oviedo

c/Julián Clavería 8, 33006-Oviedo (Spain)

E-mail: lalg@uniovi.es, vicenteruben@uniovi.es

Supporting information for this article is given via a link at the end of the document.((Please delete this text if not appropriate)) as $\mathrm{O}-\mathrm{H}$ or $1, n-\mathrm{C}-\mathrm{H}$ bond insertions, among others. ${ }^{[10]}$ Herein, we disclosed our studies on the synthesis of bifunctional allylic compounds using cyclopropenes as allyl fragment.

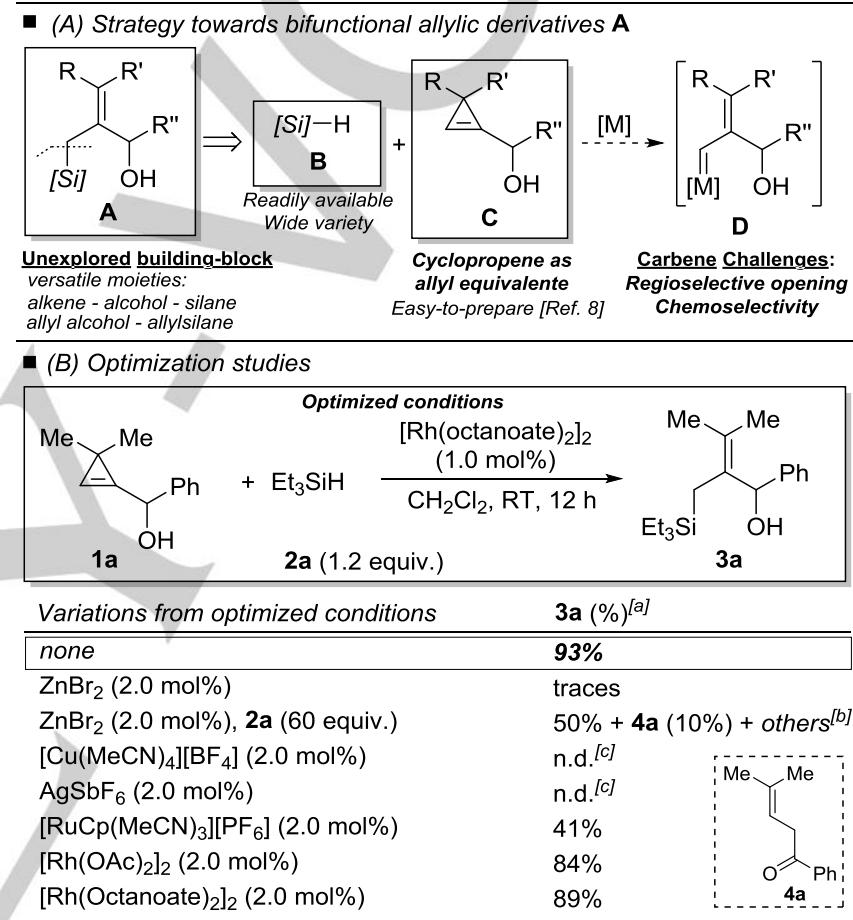

Scheme 1. Synthesis of bifunctional allylic compounds using cyclopropenes as functionalized allyl synthon: (A) Synthetic strategy. (B) Optimization studies. [a] Isolated yield. [b] Unidentified byproducts. [c] Complete degradation of 1a was observed.

To begin with, catalysts capable of promoting both cyclopropene opening to carbene and $\mathrm{Si}-\mathrm{H}$ bond insertion were considered for the model reaction of cyclopropene $\mathbf{1 a}$ with triethylsilane $(\mathbf{2 a})$ (Scheme 1, B). Disappointingly, $\mathrm{ZnBr}_{2}$ worked only when large excess of $2 \mathbf{a}$ was used (60 equiv.). ${ }^{[11]}$ Nevertheless, desired compound 3a was obtained in moderate yield $(50 \%)$, along with ketone $4 \mathrm{a}(10 \%)$ and multiple unidentified byproducts. These results indicated the challenge to control regioselectivity during carbene formation. Indeed, $\mathbf{3 a}$ arise from carbene intermediate like $\mathbf{D}$, while the formation of ketone $\mathbf{4 a}$ cannot be explained invoking this intermediate. ${ }^{[12]}$ Other archetypal transition-metal catalyst based on $\mathrm{Cu}(\mathrm{I})$ and $\mathrm{Ag}(\mathrm{I})$ led only to degradation of $\mathbf{1} \mathbf{a}^{\left[{ }^{[6]}\right.}$ Interestingly, $\left[\mathrm{RuCp}(\mathrm{MeCN})_{3}\right]\left[\mathrm{PF}_{6}\right]$ provided 3a selectively, albeit again in moderate yield (41\%). Finally, various $\mathrm{Rh}(\mathrm{II})$ carboxylate catalysts proved effective to provide $3 \mathbf{a}$ in good yield and with complete regioselectivity. In particular, the use of $\left[\mathrm{Rh}(\text { octanoate })_{2}\right]_{2}$ afforded $\mathbf{3 a}$ in a near quantitative yield (93\%) 
yield using an almost stoichiometric amount of silane 2 a (1.2 equiv.).

The scope of this reaction was subsequently examined by using optimized reaction conditions. As indicated in Scheme 2, this protocol showed a significant scope. First, we evaluated a variety of cyclopropenylcarbinol derivatives obtained from aldehydes (Scheme 2, A1), starting with those prepared from aromatic ones. Bifunctional allyl derivatives 3a-e were easily obtained in good yield (85-91\%) regardless of the electronic character of the arene. Likewise, decoration with heteroarenes such as furan or indole enabled preparation of the corresponding compounds $\mathbf{3 f}(74 \%)$ and $\mathbf{3 g}(58 \%)$, respectively. Interestingly, ferrocenyl-substituted compound $3 \mathrm{~h}$ (84\%) was cleanly prepared as well. Syntheses of compounds $\mathbf{3 i}(45 \%)$ and 3j $(77 \%)$ highlight the chemoselectivity of this transformation. Indeed, moieties reactive towards metal-carbenes (hydroxy and alkene), could be bypassed, even in an intramolecular competition event. Cyclopropenes arising from aliphatic aldehydes were then studied. Primary, secondary and tertiary alkyl substituents could be employed affording compounds $\mathbf{3 k - 0}$ in reasonable yields (71-92\%). Representative $\alpha, \beta$-unsaturated aldehydes were tested as well. Thus, using acrolein-derived cyclopropene $1 \mathrm{p}$ with dimethylphenylsilane $(\mathbf{2 c})$ led to the formation of interesting compound $3 p \quad(59 \%) .{ }^{[13]}$ Similarly, cyclohexenyl substituted derivative $\mathbf{3 q}$ could be obtained as well. In contrast, when using cyclopropene $\mathbf{1 r}$, an isomeric allylic compound $3 \mathbf{r}^{\prime}(44 \%)$ was obtained. ${ }^{[14]}$ Compounds $3 \mathbf{s} / \mathbf{s}^{\prime}$ derived from cinnamaldehyde could be selectively obtained depending on the work-up. ${ }^{[15]}$ Structure of compounds $3 \mathbf{p}$-s deserves a comment since they show not only the bifunctional $(\mathrm{OH}-\mathrm{Si})$ allyl moiety but also an additional allylic alcohol moiety, which might allow further orthogonal functionalizations. Similarly, an acetylenic moiety in cyclopropene $\mathbf{1 t}$ led to the formation of the corresponding allyl-propargyl alcohol $3 \mathbf{t}$ in a reasonable yield $(66 \%)$. It should be noticed that the protocol is scalable, as demonstrated by preparing compounds $\mathbf{3 a}$ and $\mathbf{3 0}$ at $10 \mathrm{mmol}$ scale within the same range of yields.

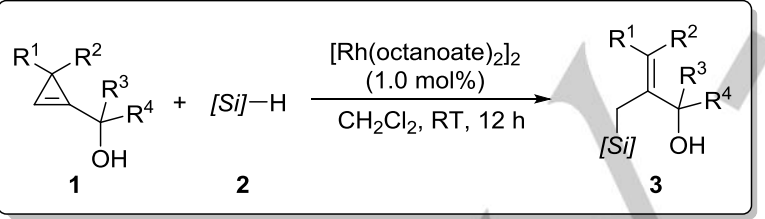

$\checkmark$ Broad scope (>35 examples) $\checkmark$ Scalable

$\checkmark$ Functional group compatibility

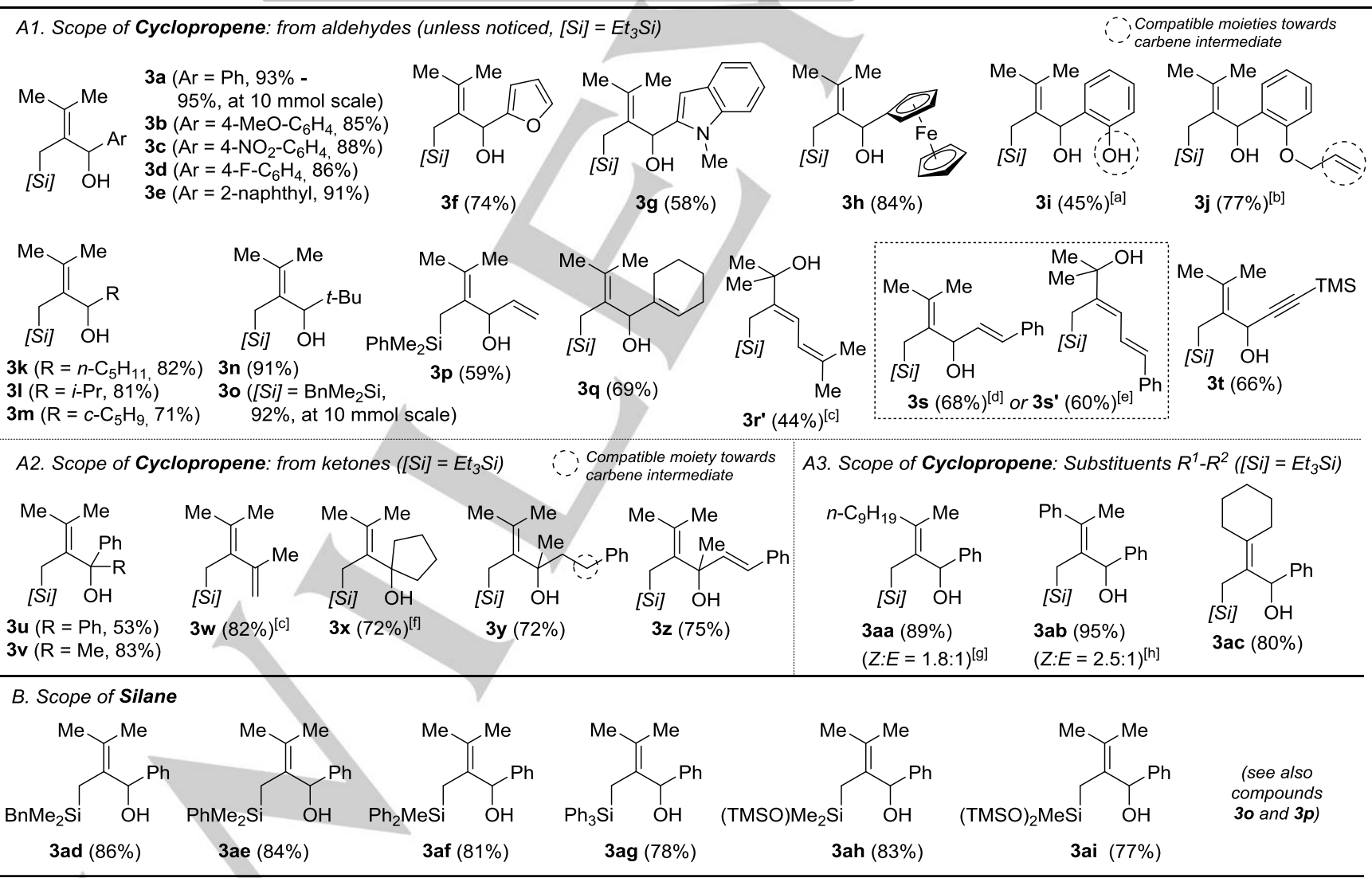

Scheme 2. Scope of $\mathrm{Rh}$ (II)-catalyzed reactions of cyclopropenes 1 with hydrosilanes 2 . Yields of isolated products; d.r. determined by ${ }^{1} \mathrm{HNMR}$ spectroscopy. [a] 3.0 equiv. of 2a were employed. [b] 20 equiv. of 2a were employed, cyclopropanation product was detected in the crude (<10\%). [c] See Ref. 14. [d] NMR-yield, 20 equiv. of $2 \mathbf{a}$ were employed. [e] After treating reaction crude with $\mathrm{SiO}_{2}$. [f] From TBDMS-protected alcohol. [g] d.r. = 1:1 of starting cyclopropene. [h] d.r. = 1:2 of starting cyclopropene. 
Studies with representative ketone-derived cyclopropenylcarbinoles 1u-z were then undertook (Scheme 2, A2). Compounds $3 \mathbf{u}, \mathbf{v}$ derived from aromatic ketones were readily obtained in $53 \%$ and $83 \%$ isolated yields, respectively. Conversely, cyclopropene $\mathbf{1 w}$ prepared from acetone led inevitably to dehydrated compound $3 w^{\prime}$ in $82 \%$ yield. ${ }^{[14]}$ On the other hand, TBDMS-protected cyclopropene $1 \mathrm{x}$ could converted directly into alcohol $\mathbf{3 x}(72 \%) .{ }^{[15]}$ The synthesis of compound $\mathbf{3 y}$ $(72 \%)$ bearing potentially reactive benzylic $\mathrm{H}$-atoms went on uneventfully, since entropically-favored $1,5-\mathrm{C}-\mathrm{H}$ insertion did not interfered. Tertiary alcohol $\mathbf{3 z}$ bearing a styryl substituent was prepared in respectable yield (75\%). Later, modification at substituents $R^{1}$ and $R^{2}$ of cyclopropene was accomplished (Scheme 2, A3). Cyclopropenes 1aa-ab, prepared and used as mixture of diastereoisomers, ${ }^{[15]}$ efficiently led to allyl derivatives 3aa,ab (89\% and $95 \%$, respectively), albeit as a mixture of $Z: E$ isomers. Symmetrically-substituted compound 3ac (80\%) was also prepared from the corresponding cyclopropene.

Taking into account the importance of the structure-reactivity relationship in silanes, ${ }^{[3]}$ the scope with respect to hydrosilanes was also accomplished (Scheme 2, B). Archetypal benzyldimethylsilane (2b), dimethylpenylsilane (2c) or diphenylmethylsilane (2d) afforded efficiently the corresponding allyl derivatives 3ad-af all in over $80 \%$ yield (see also compounds $\mathbf{3 0}, \mathbf{p})$. Sterically hindered triphenylsilane (2e) could be employed as well, giving rise to 3 ag $(78 \%)$ without erosion of the yield. A selection of allylic siloxane derivatives 3ah-ai (77$83 \%$ ), which could be later modified via cross-coupling chemistry, were also obtained with acceptable yields.

By virtue of the remarkable scope, further explorations of this reaction were accomplished. First, taking into account the importance of Si-O linkages for organic synthesis, ${ }^{[16]}$ we evaluated the feasibility of an intramolecular version of the present protocol. By conventional installation of $\mathrm{SiR}_{2} \mathrm{H}$ groups a set of hydrosilyloxicyclopropene derivatives $\mathbf{5 a - f}$ was prepared. Interestingly, by using the optimized conditions, representative cyclic siloxanes 6a-f were easily obtained in good yields (80$92 \%$, Scheme 3, A). Besides, bifunctional allyl compounds decorated with silanes and amines could be prepared as well. Here, illustrative cyclopropenes 7a-d, including usual modifications in $\mathrm{R}^{1}$ substituent (aryl, alkyl, alkenyl) or $\mathrm{N}$-atom (Ts, Boc) readily prepared from imines were employed. Pleasantly, optimized conditions led again to the corresponding aminoderivatives 8 a-d in generally good yields $(55-93 \%$, Scheme $3, B)$.

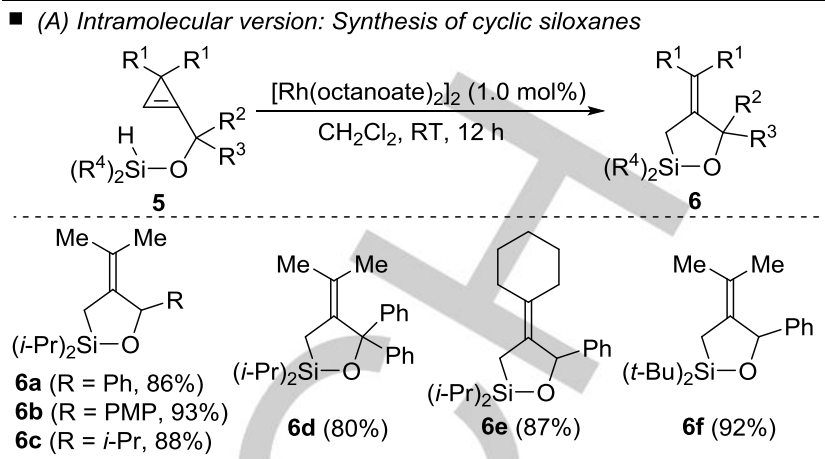

- (B) Application to amines ([Si]=Et $\left.t_{3} \mathrm{Si}\right)$

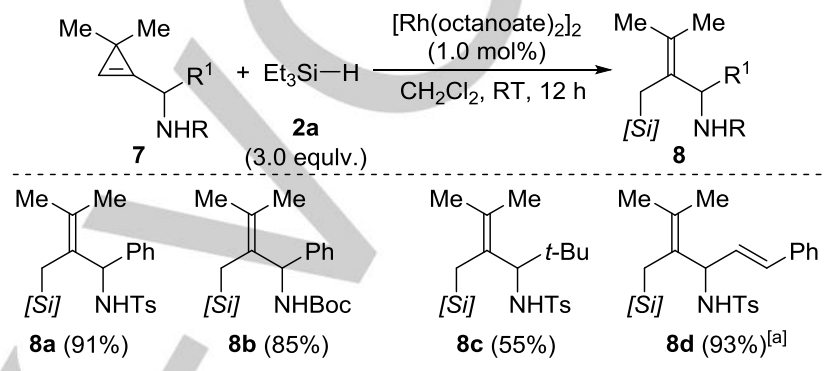

Scheme 3. Further scope: $(A)$ Synthesis of cyclic siloxanes via intramolecula reaction. (B) Application to amines. Yields of isolated products. [a] 20 equiv. of 2a were employed. $\left(\mathrm{PMP}=4-\mathrm{MeO}-\mathrm{C}_{6} \mathrm{H}_{4}\right)$.

As stated above, formation of compounds $\mathbf{3}$ might involve a rhodium-carbene intermediate generated from $\mathbf{1}^{[5,7]}$ which evolves via $\mathrm{Si}-\mathrm{H}$ bond insertion. ${ }^{[6,10]}$ However, additional interesting features were revealed by routine experiments (Scheme 4). First, the origin of the regioselectivity was explored employing cyclopropene $\mathbf{9}$, which cleanly afforded allyl silane $\mathbf{1 0}$ $(87 \%)$. This result indicates that regioselectivity strongly depends on the substitution of cyclopropene rather than on an $O$-directing effect. Thus, generation of the more substituted cyclopropyl cation I, ${ }^{[17]}$ would render $\mathrm{Rh}(\mathrm{II})$-carbene intermediate II. With respect to $\mathrm{Si}-\mathrm{H}$ bond insertion step, employment of deuterated silane [D]-2a yielded [D]-3a $(98 \%,>95 \%$ D) with complete diastereoselectivity. ${ }^{[18]}$ Moreover, an intermolecular competition experiment with equimolar amounts of $\mathbf{2 a}$ and [D]-2a provided a $k_{H} / k_{D}$ value of 2.3. This value suggests that $\mathrm{Si}-\mathrm{H}$ bond cleavage is likely involved in the RDS, in contrast with previous reports, highlighting the singularity of this transformation. ${ }^{[19]}$ The mechanism proposed also accounts for the formation of compounds 6 and 8 . 


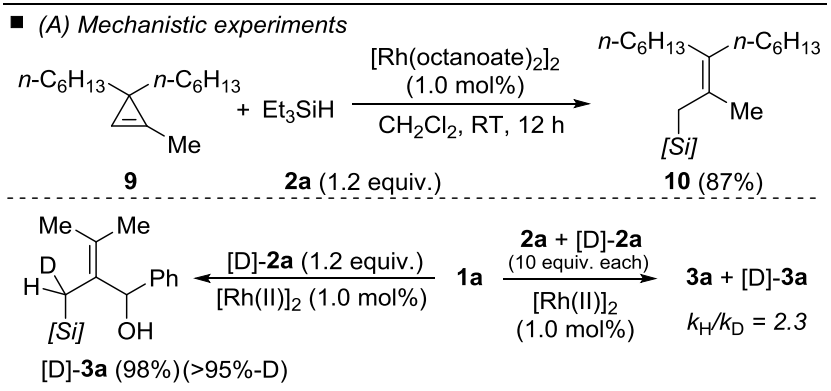

- (B) Mechanistic proposal

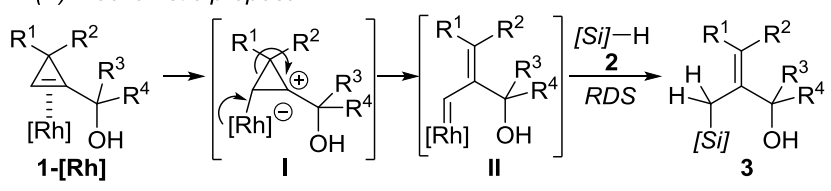

Scheme 4. Mechanistic experiments and proposal.

Finally, a set of simple, yet representative transformations of compounds $\mathbf{3}$ were performed to illustrate their synthetic utility (Scheme 5). Thus, oxidation of 3 ag with $\mathrm{MnO}_{2}$ cleanly afforded ketone 11. Treatment of 30 with $m$-CPBA led to semi-protected diol 12, likely formed via epoxidation followed by hydroxyassisted ring-opening which involved a $C$-to- $O$ silicon migration. Simmons-Smith reaction using Furukawa's protocol of $\mathbf{3 0}$ afforded diastereoselectively cyclopropane 13. Finally, fluorodesilylation of $3 \mathrm{e}$ with Selecfluor led to allyl fluoride $14 .^{[20]}$

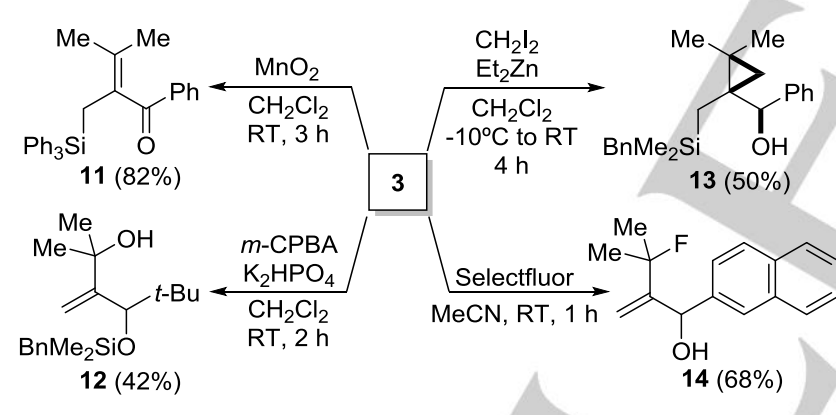

Scheme 5. Representative transformations of compounds 3. Yields of isolated products (not optimized), see the Supporting Information for detailed reaction conditions.

In summary, we have developed a method to prepare bifunctional allyl derivatives, with silane and alcohol moieties sharing the same allyl framework. This protocol relies on readily synthesizable cyclopropenes, which serve as functionalized allylic fragment source via metal vinyl carbene intermediate. A $\mathrm{Rh}$ (II)-catalyst efficiently performed the transformation, which showed a remarkable scope, including intramolecular reactions and the synthesis of bifunctional allyl compounds also with amines. These compounds might be leveraged as versatile synthetic building blocks by taking advantage of well-known chemistry of allylic compounds. Further related applications of cyclopropenes as synthetically valuable molecules are currently ongoing in our laboratories.

\section{Acknowledgements}

Financial support from Ministerio de Economía y Competitividad (MINECO), Agencia Estatal de Investigación (AEI) and Fondo Europeo de Desarrollo Regional (FEDER) (CTQ2016-76840-R) and Principado de Asturias (grant GRUPIN14-013 and Severo Ochoa PhD grant to S. M.) is gratefully acknowledged. We thank Prof. J. M. González for his support. We also thank Alba de la Fuente for the synthesis of some precursors.

Keywords: allylic compounds • alcohols • silanes • cyclopropenes $\cdot$ rhodium

[1] Selected references on the use of small molecules as synthetic building blocks, see: a) P. M. Dewick, Medicinal Natural Products: A Biosynthetic Approach, Wiley, West Sussex, UK, 2009; b) J. Li, S. G. Ballmer, E. P. Gillis, S. Fujii, M. J. Schmidt, A. M. E. Palazzolo, J. W Lehman, G. F. Morehouse, M. D. Burke, Science, 2015, 347, 1221;

[2] For a review on diversity-oriented synthesis with small molecules, see: a) W. R.J.D. Galloway, A. Isidro-Llobet, D. R. Spring, Nat. Commun 2010, 1, 80. See also: b) L. Xu, Y. Liang, Q. Ye, Z. Yang, M. Foley, S. A. Snyder, D. Ma, in Organic Chemistry, Breakthroughs and Perspectives (Eds. K. Ding, L. Dai), Wiley-VCH, Weinheim, 2012, pp. 1-31.

[3] For representative reviews on the synthetic use of allylsilanes, see: a) $\mathrm{S}$ E. Denmark, J. Fu, Chem. Rev. 2003, 103, 2763; b) J. W. J.Kennedy, D. G. Hall, Angew. Chem. Int. Ed. 2003, 42, 4732; c) L. Chabaud, P. James, Y. Landais, Eur. J. Org. Chem. 2004, 3173. See also: d) P. L. Fuchs, Handbook of Reagents for Organic Synthesis Reagents for Silicon-Mediated Organic Synthesis, Wiley-VCH, Weinheim, 2011.

[4] For representative reviews on the synthetic use of allylic alcohols and derivatives, see: a) B. M. Trost, D. L. Van Vranken, Chem. Rev. 1996, 96, 395; b) J. Muzart, Tetrahedron 2005, 4179; c) B. Sundararaju, M. Achard, C. Bruneau, Chem. Soc. Rev. 2012, 41, 4467; d) N. A. Butt. W Zhang, Chem. Soc. Rev. 2015, 44, 7929.

[5] S. Mata, L. A. López, R. Vicente, Angew. Chem. Int. Ed. 2017, 56, 7930

[6] For selected examples of metal-catalyzed insertion of metal-vinyl carbenes into Si-H bond, see: a) J. Wu, Y. Chen, J. S. Panek, Org. Lett. 2010, 12, 2112; b) J. Wu, J. S. Pakek, J. Org. Chem. 2011, 76, 9900; c) D. Chen, D.-X. Zhu, M.-H. Xu, J. Am. Chem. Soc. 2016, 138, 1498. For a review on synthetic applications of metal carbenes generated from cyclopropenes, see: a) A. Archambeau, F. Miege, C. Meyer, J. Cossy, Acc. Chem. Res. 2015, 48, 1021. See also: b) R. Vicente, Synthesis 2016, 2343.

[8] A scalable two-step synthesis of cyclopropenes such as $\boldsymbol{C}$, has been described: a) F. Miege, C. Meyer, J. Cossy, Org. Lett. 2010, 12, 4144 b) F. Miege, C. Meyer, J. Cossy, Chem. Eur. J. 2012, 18, 7810; c) S Simaan, A. Masarwa, E. Zohar, A. Stanger, P. Bertus, I. Marek, Chem. Eur. J. 2009, 15, 8449 .

[9] For reviews on cyclopropene chemistry, see: a) Z.-B. Zhu, Y. Wei, M Shi, Chem. Soc. Rev. 2011, 40, 5534; b) M. Rubin, M. Rubina, V. Gevorgyan, Chem. Rev. 2007, 107, 3117; c) I. Marek, S. Simaan, Samah, A. Masarwa, Angew. Chem. Int. Ed. 2007, 46, 7364 See also Ref. 7.

[10] a) M. P. Doyle, Chem. Rev. 1986, 86, 919; b) F. Z. Dörwald (Ed.) Metal Carbenes in Organic Synthesis, Wiley-VCH, Weinheim, 1999.

[11] The use of less excess of $2 \mathbf{a}$ (1.2 to 20 equiv.) led to unmanageable reaction mixtures, in which $3 \mathbf{a}$ could be detected (typically $<10 \%$ ).

[12] Ketone 4 a could likely be formed via regioisomeric carbene intermediate ' D', followed by a $1,2-\mathrm{C}-\mathrm{H}$ insertion and keto-enol tautomerization.

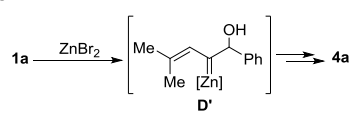


[13] The corresponding compound derived from the reaction with $\mathrm{Et}_{3} \mathrm{SiH}$ (2a) could not be isolated.

[14] An in-situ isomerization and dehydration might account for the formation of $3 r^{\prime}$ and $3 w^{\prime}$, respectively.

[15] See the Supporting Information for details

[16] a) M. N. Missaghi, J. M. Galloway, H. H. Kung, Organometallics 2010 29, 3769; b) S. E. Denmark, C. S. Regens, Acc. Chem. Res. 2008, 41 1486. See also Ref. $2 d$.

[17] This regiochemical outcome is in good agreement with earlier findings, see Ref. 5, 7a and 8a. For a seminal contribution on regioselective generation of rhodium carbene intermediates from cyclopropenes, see:
P. Müller, N. Pautex, M. P. Doyle, V. Bagheri, Helv. Chim. Acta, 1990 73, 1233.

[18] The relative configuration could not be determined, see the Supporting Information for details.

[19] For a representative example, see: L. A. Dakin, P. C. Ong, J. S. Panek, R. J. Staples, P. Stavropoulos, Organometallics 2000, 19, 2896. See also Ref. 5.

[20] Reaction conditions were described in: a) Y. Landais, L. Parra-Rapado, Tetrahedron Lett. 1996, 37, 1205 (alkene oxidation); b) A. B. Charette, H. Lebel, J. Org. Chem. 1995, 60, 2966 (cyclopropanation); c) B Greedy, J. M. Paris, T. Vidal, V. Gouverneur, Angew. Chem. Int. Ed. 2003, 42, 3291 (fluorodesilylation). 


\section{Entry for the Table of Contents}

\section{COMMUNICATION}

$$
\begin{aligned}
& R^{1} R^{2} R^{1} R^{2} \quad \checkmark \text { New Bifunctional allyl derivatives (over } 40 \text { examples) }
\end{aligned}
$$

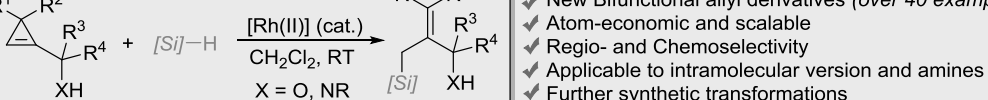

Synthesis of uncommon bifunctional allylic derivatives bearing a silane and an alcohol within the same allylic system is disclosed. The method relies on the use of hydrosilanes and adequately functionalized cyclopropenes, which deliver the allyl fragment by regioselective generation of a $R h(I I)$ vinyl carbene intermediate. The protocol shows a remarkable broad scope, including intramolecular version for the synthesis of cyclic siloxanes or extension to allylic amines.
Sergio Mata, Luis A. López*, Rubén Vicente* $^{*}$

Page No. - Page No.

Synthesis of Bifunctional Allylic Compounds using Cyclopropenes as Functionalized Allyl Equivalents 